\title{
A Methodology for Validating Artifact Removal Techniques for fNIRS
}

\author{
Kevin T Sweeney, Hasan Ayaz, Tomás E Ward, Meltem Izzetoglu, Seán F McLoone and Banu Onaral
}

\begin{abstract}
NIRS recordings are increasingly utilized to monitor brain activity in both clinical and connected health settings. These optical recordings provide a convenient measurement of cerebral hemodynamic changes which can be linked to motor and cognitive performance. Such measurements are of clinical utility in a broad range of conditions ranging from dementia to movement rehabilitation therapy. For such applications fNIRS is increasingly deployed outside the clinic for patient monitoring in the home. However, such a measurement environment is poorly controlled and motion, in particular, is a major source of artifacts in the signal, leading to poor signal quality for subsequent clinical interpretation. Artifact removal techniques are increasingly being employed with an aim of reducing the effect of the noise in the desired signal. Currently no methodology is available to accurately determine the efficacy of a given artifact removal technique due to the lack of a true reference for the uncontaminated signal. In this paper we propose a novel methodology for fNIRS data collection allowing for effective validation of artifact removal techniques. This methodology describes the use of two fNIRS channels in close proximity allowing them to sample the same measurement location; allowing for the introducing of motion artifact to only one channel while having the other free of contamination. Through use of this methodology, for each motion artifact epoch, a true reference for the uncontaminated signal becomes available for use in the development and performance evaluation of signal processing strategies. The advantage of the described methodology is demonstrated using a simple artifact removal technique with an accelerometer based reference.
\end{abstract}

\section{INTRODUCTION}

Functional Near Infrared Spectroscopy (fNIRS) is an increasingly popular optical technique for monitoring cerebral hemodynamic changes during brain activity. As individual regions of the brain are activated, due to internal or external stimuli, there are concomitant changes in the levels of oxyhemoglobin (oxy-Hb) and deoxy-hemoglobin (deoxy-Hb) concentrations in the area. These hemodynamic changes affect the optical absorption properties of the region. fNIRS employs light at different wavelengths (in the visible to near infrared range) to penetrate the skull and indirectly obtain a measurement for the change in concentration levels of both oxy-Hb and deoxy- $\mathrm{Hb}$ [1] through changes in light absorption. By determining this shift in the levels of oxy$\mathrm{Hb}$ and deoxy-Hb, activation changes within the cortex can

This work was supported in part by Irish Research Council for Engineering, Science and Technology (IRCSET) and Science Foundation Ireland: Research Frontiers Program 2009, Grant No. 09/RFP/ECE2376

K.T. Sweeney, T.E. Ward and S.F McLoone are with the Department of Electronic Engineering, National University of Ireland Maynooth, Co. Kildare, Ireland. email: ksweeney @eeng.nuim.ie

H. Ayaz, M. Izzetoglu and B. Onaral are with the School of Biomedical Engineering, Science \& Health Systems, Drexel University, Philadelphia, PA, USA. email: ayaz@drexel . edu be determined.

fNIRS has been implemented in a number of diverse areas including human performance assessment [2], motor tasks [5] and Brain-Computer Interfaces (BCI) [6]. fNIRS technology can provide a variety of advantages compared to other established neuroimaging modalities. For example, fNIRS offers superior spatial resolution, as compared to electroencephalography (EEG), it is more affordable, in comparison to functional magnetic resonance imaging (fMRI) and it is noninvasive as compared to positron emission tomography (PET). Furthermore, since the fNIRS technology measures the changes in the optical properties of the tissue, it is not susceptible to artifacts of electrophysiological nature such as the signals associated with eye movement or muscular contractions.

Although not as pronounced as in other neuroimaging modalities, such as fMRI, motion artifacts still pose a challenge to the analysis of fNIRS measurements. This motion artifact can manifest itself in two distinct ways. Firstly, it can be due to subject motion, where movement of the subject causes changes in the recorded blood volume in the cortical area of interest. This increase/decrease in blood volume does not account for a change in the true activation level in the cortex and is thus considered as an artifact. Secondly, movement of the optode with respect to the skin can cause a sudden change in recorded light intensity. This variation can be due to a change in the path length (distance the light must travel from the source to the detector), or alternatively can be due to the detector picking up light from an unwanted external light source or the direct light reflected from the skin. The external light can fall on the optical sensor because the optical coupling between the skin and then optode has been physically compromised resulting in the admission of external light sources to the measurement process.

There exist a number of signal processing techniques and algorithms in the literature for the removal or reduction of motion-induced artifacts in fNIRS. Adaptive filters have been utilized to remove the unwanted noise [4] [8] and have been shown to produce a moderate improvement in signal to noise ratio (SNR). Izzetoglu et al also proposed Wiener [4] and discrete Kalman filtering [3] techniques for the removal of motion artifacts. Wiener filtering has been observed to produce a greater improvement in SNR than that realized using adaptive filtering methods while also reducing the need for additional sensors to capture some measure of the noise source. However the Wiener filter requires the pre-processing to be done off-line. The Kalman filter had comparable SNR improvement to that observed using the 
Wiener filter however it is capable of being used in real-time operation thus adding an additional advantage. Robertson et $a l$ and Molavi et al utilized the wavelet transform to remove motion artifact in [8] and [7] respectively. The wavelet transform was found to be useful when the artifact was in the form of sudden movements or spikes, however the off-line requirement of the algorithm is a disadvantage.

One major predicament faced by all researchers, when trying to remove any form of motion artifact from the desired signal, is the lack of knowledge of the true form of the original (noise-free) signal prior to the addition of the artifact. Currently this can only be achieved in fNIRS through simulation. Without knowledge of this original signal it is difficult to accurately determine the efficacy of a given motion artifact removal technique. In this paper we propose a source-detector configuration and a methodology to obtain both the noise-free and noisy fNIRS recording concurrently. The method uses two fNIRS channels situated in close proximity. Accelerometers are also employed to determine when motion has occurred and are used as a noise reference in the adaptive filter application as described in Section III. The measurements collected with the described methodology suggest that the source detector configuration effectively provides a noiseless signal while concurrently providing a noisy, motion artifact contaminated, signal. Consequently the methodology allows the acquisition of signal sets ideally suited for the design, test and validation of motion artifact removal methods for fNIRS.

As an example of the utility of the methodology we have applied a motion artifact removal technique, namely adaptive filtering, to the data collected from the proposed system. The adaptive filtering results suggest that the noise within the fNIRS recordings can be adequately removed tending the cleaned signal towards the noiseless fNIRS recording demonstrated by an increase in SNR and cross correlation coefficient.

The remainder of the paper is structured as follows: Section II describes the experimental setup and procedure used in this paper, Section III presents example recordings of noise-free and noisy fNIRS recordings where the advantage of the proposed experimental setup in the performance assessment of motion artifact removal is demonstrated through the use of adaptive filtering algorithm. Finally the conclusion and future work are discussed in Section IV.

\section{EXPERIMENTAL SETUP}

\section{A. Data Acquisition System}

fNIRS and accelerometer data containing epochs of motion and motion-free recordings was collected during 5 sessions from two male subjects. The fNIRS data was collected using a TechEn CW6 system (TechEn Inc., USA) at a sampling frequency of $25 \mathrm{~Hz}$. The system uses source wavelengths of $690 \mathrm{~nm}$ and $830 \mathrm{~nm}$. Two tri-axial ADXL327 accelerometers (Analog Devices) were also utilized in the experiment to determine the time, magnitude and direction of motion. The accelerometer outputs are sampled at $2048 \mathrm{~Hz}$ and have a full scale range of $\pm 2 \mathrm{~g}$.
The relative positions of the fNIRS optodes and the accelerometers as used during experimentation is illustrated in Fig. 1.

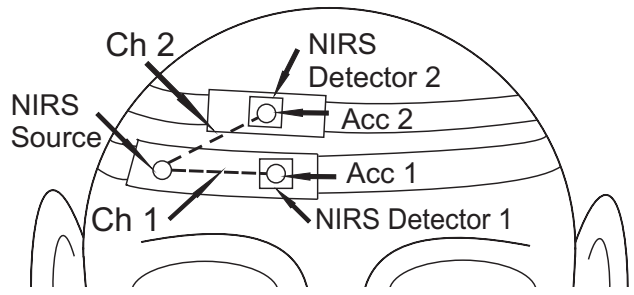

Fig. 1. Position of the NIRS optodes and accelerometers on the right prefrontal cortex during experiment. Channel 1: Positions are fixed. Channel 2: Channel path perturbed due to movement of Detector 2

The fNIRS source optode is connected to a plastic housing which is secured to the head using the lower head-strap. The plastic housing is comprised of low-density polythene backed by polyurethane foam. This plastic housing also encompasses one of the two separate detectors (Detector 1) utilized in the experiment. The second detector is connected to the head using a separate housing and head-strap and is positioned to ensure the same source detector spacing, of $3 \mathrm{~cm}$, is retained. The two accelerometers are connected directly to the individual detectors and are adjusted to ensure the axes are properly aligned with respect to each other. It should be noted that the two pairs of detectors and accelerometers are not directly coupled together, and therefore movement of one detector has little or no influence on the position of the second. All post-processing was performed in off-line mode using MATLAB (2008b, The MathWorks, Natick, MA).

\section{B. Experimental Procedure}

The fNIRS and accelerometer systems were set up as described and shown in Section II-A. The source detector pairs were positioned to record from the right pre-frontal cortex. The objective of the performed experiments was to display the difference between motion-contaminated data and motion-free data; therefore the underlying cortical activity was not of significance for this study. Furthermore, recording was not performed from other areas on the scalp so as to eliminate the possibility of hair blocking the contact of the optode with the scalp both before and after movement.

As the underlying cortical activity is not of importance for this paper, the subjects were not asked to perform any specific activity during the duration of the experiment. Due to the close proximity of the two channels, the underlying cortical activity is assumed to be similar, producing similar recordings. Each recording session lasted 9 minutes. At regular 1 minute intervals the experimenter induced a positional disturbance to Detector 2 attached to Accelerometer 2. This source detector pairing was labeled Channel 2. This slight disturbance, which was performed manually, induced motion artifact on Channel 2. However, as Detector 1, connected to Accelerometer 1, was not disturbed; Channel 1 remained free of contamination throughout the duration of the experiment. This result can be observed in Fig. 2. 

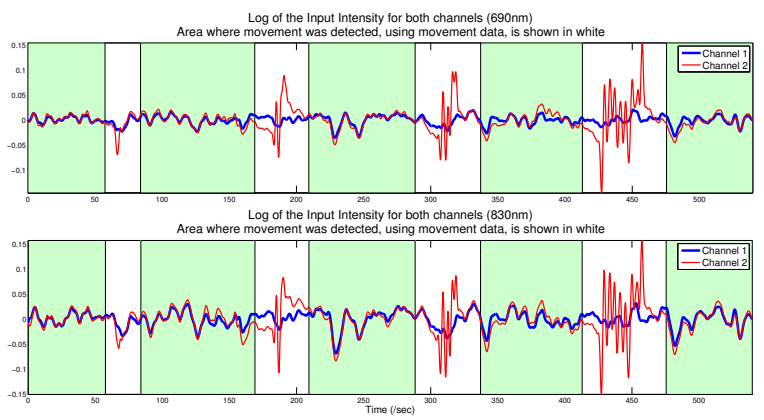

Fig. 2. Log of the input intensities recorded using $690 \mathrm{~nm}$ and $830 \mathrm{~nm}$ wavelengths. Shaded section contains no motion artifact as determined using the accelerometers.

The sections highlighted with a shaded area are epochs of motion free data where Channel 2 follows the output of Channel 1 closely. However, during times of motion, determined by the differential movement of the accelerometers, Channel 2 can be observed to contain motion artifacts.

\section{RESULTS \& DISCUSSION}

The "one source, two detector" experimental setup, described in Section II, allows for the recording of two separate fNIRS channels, one clean and the other occasionally contaminated with motion artifact. As stated previously, the two detectors are in close proximity, therefore their corresponding measurement volumes within the brain (between source and detector) overlap significantly and the channels are thus expected to record the same, or similar, true brain signal. However, when motion artifact is induced in one detector, as described in Section II-B, the signal can differ greatly depending on the magnitude of the artifact (Fig. 2). We performed a comparison of Channel 1 and Channel 2 recordings for epochs where there is no motion artifact and when the motion contamination is present based on correlation analysis. The two channel recordings are very similar with a high correlation $(\mathrm{R}=0.777)$ when no artifact is induced (as shown by shaded areas in Figure 2). However, when motion artifact is induced, comparison of the channels yields much lower correlation $(\mathrm{R}=0.358)$ as expected.

Adaptive filtering has been widely used as an optimal noise removal algorithm in various biomedical applications. This technique requires simultaneous measurements of the correlated motion artifact signal from a separate sensor which is usually an accelerometer. In this study we have used accelerometer data as reference signal and the light intensity time-series as the primary input signal. Details of the technique can be found in [4]. To illustrate the utility of the methodology, a normalized least mean squares (NLMS) adaptive filter has been employed in this study to clean the motion artifact contaminated epochs (recorded by Channel 2). Here, the NLMS algorithm is chosen due to its computational simplicity and to guarantee stability. The primary input for the adaptive filtering application, consisting of the true signal plus noise, is obtained from the Channel 1 measurement. The reference signal is obtained from the accelerometer recordings (Acc 2 as shown in Figure 1). This signal is down-sampled to the same sampling frequency, of $25 \mathrm{~Hz}$, as the fNIRS recordings. The filter coefficients of the NLMS filter are updated at each iteration using the primary and reference inputs. An estimate of the noise-free true signal is then obtained at the output. The output signal estimates are then compared with the known true (motion-free) signals recorded by Channel 1 for performance evaluation. Note that the motion artifact, as implemented during the overall course of the protocol used in this study, is non-stationary in nature. Adaptive filters can be successfully applied for the cancelation of noise of a stationary as well as a non-stationary nature due to its capability to adapt the filter parameters to changing signal characteristics. However, in this study the adaptive filter algorithm is applied only to segments of data where motion artifact is known to be present. Within these segments the noise can be assumed to be stationary since we are merely interested in the performance of the algorithm in the suppression of the motion artifact. The noisy segments of data are extracted using the accelerometer recordings.

An example noise reduced signal as estimated by the adaptive filter together with the noisy (Channel 2) and noisefree (Channel 1) fNIRS recordings and the correlated noise reference (accelerometer data) are shown in Fig. 3. As can be seen from this example epoch the adaptive filter was able to effectively suppress motion artifact resulting in a cleaned signal estimate that follows a similar pattern to that of the true motion-free signal.

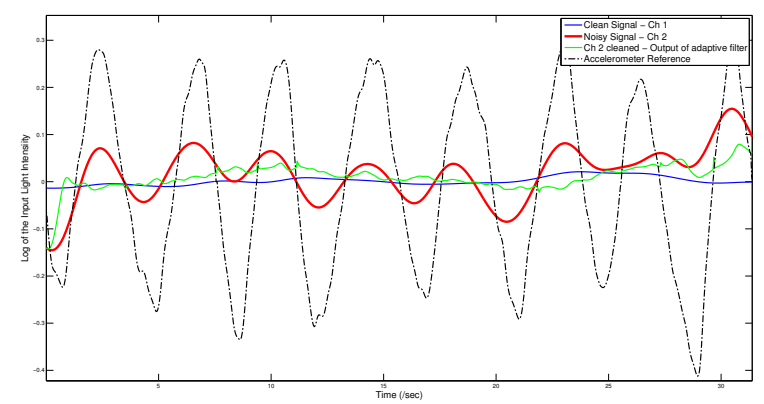

Fig. 3. Example of artifact removal using an NLMS Adaptive Filter.

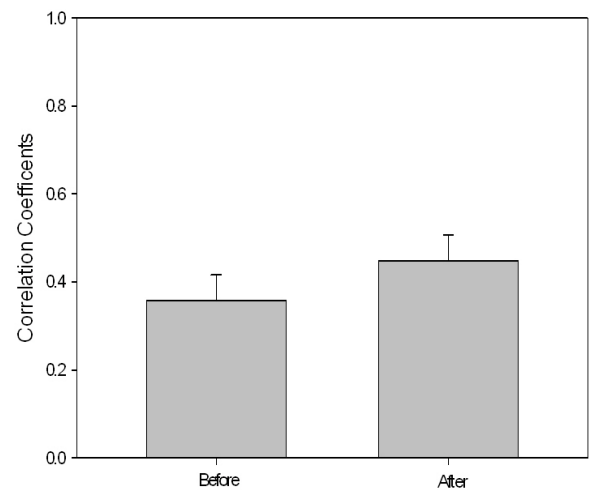

Fig. 4. Correlation coefficients of noisy epochs (before adaptive filtering) and cleaned epochs (after adaptive filtering). Error bars are SEM. 
TABLE I

STATISTICAL COMPARISON OF BEFORE VS. AFTER EPOCHS

(TWO-TAILED PAIRED T-TESTS; DF=20)

\begin{tabular}{|c|c|c|c|c|c|}
\hline & $\mathbf{t}$ & $\mathbf{p}$ & $\begin{array}{c}\text { Mean } \\
\text { Diff }\end{array}$ & $\begin{array}{c}\text { Lower Limit } \\
\text { \%95 CI }\end{array}$ & $\begin{array}{c}\text { Higher Limit } \\
\text { \%95 CI }\end{array}$ \\
\hline SNR & 7.177 & $<0.001$ & 5.935 & 4.209 & 7.660 \\
\hline CC & 3.585 & $<0.01$ & 0.09 & 0.038 & 0.143 \\
\hline
\end{tabular}

There was a total of 21 motion artifact epochs in 5 different recording sessions from two subjects, determined using the accelerometer data. Adaptive filtering is solely applied to these 21 individual, available, epochs of data. In order to quantitatively analyze the performance of the adaptive filter in the suppression of motion artifacts, we use two measures based on SNR and correlation coefficient (CC). The SNR in this study was calculated using the following formula:

$$
S N R=10 \log _{10}\left(\frac{\sigma_{x}^{2}}{\sigma_{e}^{2}}\right)
$$

where $\sigma_{x}^{2}$ is the variance of the clean noise-free signal (Channel 1) and $\sigma_{e}^{2}$ is the variance of the error signal. The error signal before the application of adaptive filtering is the motion artifact found by subtracting the noise-free (Channel 1) recording from the noisy (Channel 2) measurement, assuming that motion artifact is additive. After the application of the adaptive filtering the estimation error signal is determined by subtracting the noise-free signal from the cleaned signal obtained as a result of the adaptive filtering operation. The SNR is individually calculated over the 21 epochs of noisy data. In CC analysis, before the application of adaptive filtering, the $\mathrm{CC}$ is obtained between the epochs of actual noise-free and noisy recordings and after the application of adaptive filter, the CC is calculated between the actual noise-free and the cleaned signal obtained as the output of the adaptive filter.

The average SNR of the original noisy signal epochs was calculated to be $-14.37 \mathrm{~dB}$. After employing the adaptive filter this SNR value rose by $5.93 \mathrm{~dB}$ to $-8.44 \mathrm{~dB}$. Figure 4 illustrates the $\mathrm{CC}$ between the known true signal and the noisy epochs and cleaned epochs (after applying adaptive filter) respectively. Although the adaptive filter provided significant improvements in both SNR and CC, comparing the clean epoch correlation $(\mathrm{R}=0.777)$ to the cleaned epoch correlation after the adaptive filter $(\mathrm{R}=0.448)$ illustrates the requirement for more powerful artifact removal techniques.

This test methodology, for the first time, allowed for a better comparison and performance evaluation of a motion artifact removal algorithm by providing a good estimate of the true noise-free signal as a reference. Statistical comparison of the SNR and CC with paired t-test indicates significant improvement for both as reported in table I.

\section{CONCLUSION AND FUTURE WORK}

In this study a source detector configuration, in conjunction with a motion artifact generation methodology, is proposed for fNIRS applications which can be employed in the comparison of motion artifact removal techniques. Previous studies have been un-able to accurately determine the efficacy of the numerous artifact removal techniques due to the lack of knowledge of the true noise-free signal. The proposed new methodology is capable of producing noiseless-true fNIRS measurements in conjunction with motion artifact corrupted recordings, thus providing an accurate reference for the true signal. In this paper we have analyzed motion artifact resulting from movement of the fNIRS sensor and concurrently evaluated the efficacy a single removal technique. It is shown that the recordings obtained, through the proposed configuration, can be effectively used in the performance analysis of a basic motion artifact removal algorithm, adaptive filtering.

Future work will incorporate a performance comparison of different artifact removal techniques (including Wiener and Kalman filtering) using the measurements obtained through the proposed methodology while subjects are resting and performing a cognitive task. In addition, a study will be conducted to determine the motion artifact introduced by the movement of the head itself. In this latter study it can be argued that motion artifact can exist in both detector recordings which can, in fact, be the situation in most real-life applications. In such instances, both the motion artifact and the brain signals will exist simultaneously in both detectors, thus blind source separation techniques such as principal component analysis (PCA) or independent component analysis (ICA) based methods may be effectively employed which will also be studied as a future work.

\section{REFERENCES}

[1] S.C. Bunce, M. Izzetoglu, K. Izzetoglu, B. Onaral, and K. Pourrezaei. Functional near-infrared spectroscopy. Engineering in Medicine and Biology Magazine, IEEE, 25(4):54-62, July-Aug. 2006.

[2] K Izzetoglu, S Bunce, B Onaral, K Pourrezaei, and B Chance. Functional Optical Brain Imaging Using Near-Infrared During Cognitive Tasks. International Journal of human-computer interaction, 17:211227, 2004.

[3] M. Izzetoglu, P. Chitrapu, S. Bunce, and B. Onaral. Motion artifact cancellation in NIR spectroscopy using discrete Kalman filtering. BioMedical Engineering OnLine, 9(1):16, 2010.

[4] M. Izzetoglu, A. Devaraj, S. Bunce, and B. Onaral. Motion artifact cancellation in NIR spectroscopy using Wiener filtering. Biomedical Engineering, IEEE Transactions on, 52(5):934-938, May 2005.

[5] D.J. Leamy and T.E. Ward. A novel co-locational and concurrent fNIRS/EEG measurement system: Design and initial results. In Engineering in Medicine and Biology Society (EMBC), 2010 Annual International Conference of the IEEE, pages 4230 -4233, 312010-sept.4 2010.

[6] F. Matthews, B.A. Pearlmutter, T.E. Ward, C. Soraghan, and C. Markham. Hemodynamics for Brain-Computer Interfaces. Signal Processing Magazine, IEEE, 25(1):87 -94, 2008.

[7] B. Molavi, G. Dumont, and B. Shadgan. Motion artifact removal from muscle NIR Spectroscopy measurements. In Electrical and Computer Engineering (CCECE), 2010 23rd Canadian Conference on, pages 1 -4, May 2010.

[8] F. C. Robertson, T. S. Douglas, and E. M. Meintjes. Motion Artifact Removal for Functional Near Infrared Spectroscopy: A Comparison of Methods. Biomedical Engineering, IEEE Transactions on, 57(6):1377 -1387 , June 2010 